\title{
Literaturverzeichnis
}

[1] G. Birkhoff, Lattice Theory, rev. ed. New York 1948.

[2] N. Bourbaki, Topologie générale, Eléments de Mathématique II, première partie Les struetures fondamentales de l'analyse, livre III, Paris 1940.

[3] E. Čech, Topologické prostory, Nakl. ČSAV, Praha 1959.

[4] - Topologické prostory, Čas. pěst. mat. a fys. 66 (1937) S. D 225-264.

[5] M. Norotný, Bemerkung über die Darstellung teilweise geordneter Mengen, Publ. Fac. Sci. Univ. Masaryk, Brno, 369 (1955), S. 451-458.

\section{Some applications of the notions of forcing and generic sets *}

by

\section{S. Feferman (Stanford, Calif.)}

1. The notions of forcing and of generic sets were introduced by Paul Cohen [2], [3] to settle the long-outstanding problems of the logical interrelationships of the axiom of constructibility, the axiom of choice, and the continuum hypothesis, relative to the system of Zermelo-Fraenkel set theory. In this paper we consider extensions of these notions to other contexts, namely that of (1st order) number theory and of a part of (2nd order) analysis, and obtain some applications there ( $\$ 2$ and 3). These results depend on a general transform lemma concerning forcing; this is proved in $\S 2$ below. By means of this lemma we are also able to obtain some new applications of Cohen's methods in set theory $(\S 4)$. The most interesting of these are the following: (1) No set-theoretically definable well-ordering of the continuum can be proved to exist from the Zermelo-Fraenkel axioms together with the axiom of choice and the generalized continuum hypothesis. (2) The prime ideal theorem in Boolean algebra is independent of the Zermelo-Fraenkel axioms. (Both results depend, of course, on the hypothesis of consistency of ZermeloFraenkel set theory.)

The notion of forcing is syntactic and the notion of generic (sequence of) sets is obtained directly from it. However, the motivations behind the introduction of these notions are essentially model-theoretic. In broad terms, what is involved is the following. One starts with a certain language $\mathrm{L}$, whose structures $\mathcal{H}$ we are interested in. $\mathrm{L}$ is extended to an auxiliary language $\mathrm{L}^{*}=\mathrm{L}^{*}\left(\mathbf{S}_{0}, \ldots, \mathbf{S}_{n}, \ldots\right)$ containing (a finite or infinite sequence of) symbols $\mathbf{S}_{0}, \ldots, \mathbf{S}_{n}, \ldots, n<\delta \leqslant \omega$, for the generie sets $S_{0}, \ldots, S_{n}, \ldots$ to be defined. $\mathrm{L}^{*}$ also contains means to denote the members of a structure $\mathcal{H}^{*}=\mathcal{H}^{*}\left(S_{0}, \ldots, S_{n}, \ldots\right)$ of objects constructed in certain ways from the $S_{0}, \ldots, S_{n}, \ldots$ Every $\mathrm{L}^{*}$-structure thus determines an $\mathrm{L}$-structure.

* Text of a talk given under this title at the International Symposium on the Theory of Models held at Berkeley, June 25-July 11, 1963. A summary of the main definitions and results of this paper is to appear under the same title in the proceedings of that Symposium.

Fundamenta Mathematicae, T. LVI 
The definition of forcing is given inductively for sentences of $L^{*}$. Intuitively speaking, a sentence $\mathfrak{F}$ of $L^{*}$ is forced by a finite amount of information $Q$ about the members of the sets $S_{0}, \ldots, S_{n}, \ldots$, if the truth of $\mathfrak{F}$ in $\mathcal{H}^{*}\left(S_{0}, \ldots, S_{n}, \ldots\right)$ can be established on the basis of this information and will remain established no matter how $Q$ is extended. We cannot, in general, expect for any given $S_{0}, \ldots, S_{n}, \ldots$ and any sentence $\mathfrak{F}$ of $\mathrm{L}^{*}$, that the trath of $\mathfrak{F}$ or $\sim \widetilde{F}$ in $\mathscr{K}^{*}$ can be determined in this way, i.e. that some finite amount of information $Q$ forces $\widetilde{F}$ or forces F. Those sequences for which this does hold are said to be generic. Then, in accordance with the intuitive ideas which led to the definition of forcing, Cohen's basic theorem shows that if $S_{0}, \ldots, S_{n}, \ldots$ is a generic sequence and $\widetilde{F}$ is a sentence of $L^{*}$ then $\widetilde{F}$ is true in $\mathcal{K}^{*}$ if and only if $\xi$ is forced by some finite amount of information about the members of the sets $S_{0}, \ldots, S_{n}, \ldots$ At the same time this result provides a reduction of the determination of various properties of $\mathcal{H}^{*}$ (and its induced $\mathrm{L}$-structure) to syntactic questions about forcing. $\mathrm{It}$ is from this theorem that all the applications flow, once the existence of generic sequences is established.

In the simplest of the cases taken up in Cohen [2], $\mathrm{L}$ is the language of set theory and $\delta=1$; in other words, only a single symbol $\mathbf{S}\left(=\mathbf{S}_{0}\right)$ is adjoined in this case. Let $\alpha_{0}$ be a denumerable ordinal such that the structure $\mathcal{H}$ of sets constructible in less than $\alpha_{0}$ steps, in the sense of Gödel [8], forms a model of Zermelo-Fraenkel set theory. Then $L^{*}(\mathbf{S})$ also contains constant symbols $\mathrm{F}_{\alpha}(\mathbf{S})$ and ranked variable symbols $\mathbf{X}^{a}, Y^{a}, \ldots$ for each $a<\alpha_{0}$. The intention is that $\mathcal{H}^{*}(S)$ shall consist of the sets $F_{\alpha}(S)$ constructible from $S$ in $\alpha$ steps, $\alpha<\alpha_{0}$, and that the range of the ranked variables $\mathrm{X}^{\alpha}$ shall be $\left\{F_{\beta}(S): \beta<\alpha\right\}$. The definition of forcing is given by Cohen in two stages: first, for what he calls limited statements, in which all variables are ranked, and then by extension to arbitrary statements of $\mathrm{L}^{*}$. In both cases, however, he restriets the definition to statements in prenex normal form. By means of the reduction of truth in $\aleph^{*}$ to forcing in $\mathrm{L}^{*}$, Cohen shows that for generic $S, \mathcal{H}^{*}(S)$ is a model of Zermelo-Fraenkel set theory together with the axiom of choice and the generalized continuum hypothesis, but relative to which $S$ is not constructible.

Dana Scott made several suggestions to us for treating the notions considered here in a way which would be more readily adaptable to a variety of languages and which would make the general development smoother going (1). These were as follows. First of all, he suggested that the language $L^{*}$ to be used in the case of set theory be modified so as

(1) We are greatly indebted to Professor Scott for these suggestions, as will be seen from what follows. We also wish to thank him for his useful comments on a draft
of this paper. to correspond instead to the original definition of constructibility introduced in Gödel [7]; this was given in ramified terms rather than in terms of the specific sequence of constructions $F_{a}$. (Among other advantages, this aroids having to re-determine the initial values of the sequence $F_{\alpha}(S)$ according to different applications, as was necessary in Cohen's work.) Second, he showed us a simple way to define the notion of forcing for arbitrary sentences of $\mathrm{L}^{*}$, without restriction to prenex normal form. (This definition does not coincide exactly with Cohen's on prenex sentences, but it has the same desired main properties.) Finally, he pointed out that a closely related definition of forcing could be given for a number-theoretic language $L^{*}$, again with interesting properties.

Our own interest in this subject began with the idea of applying Cohen's methods to the construction of models of hyperarithmetic analysis. Scott's suggestions turned out to be extremely useful to us, for we realized that they could also be adapted to this situation. Our work in this area then led us to see that they could be used to give a more general treatment which would have applications in number theory, analysis, and set theory. Namely, the definition of forcing and its main properties can be developed for an auxiliary language $L^{*}$ framed in the ramified theory of types, including variables $\mathrm{X}^{\alpha, \beta}$ of (possibly transfinite) type $\beta<\beta_{0}$ and ramification rank $a<\alpha_{0}$. The specific cases are dealt with by choosing different values for $\alpha_{0}, \beta_{0}$. (However, for the special case of set theory it is simpler to use a ramified type-free language, as in Gödel [7].)

For the purposes of exposition, we do not present this general approach here. Rather, we begin in the next section with a definition of forcing for the simplest case, $\beta_{0}=1, \alpha_{0}=0$. We then show how the definition is to be extended in each of the succeding special cases for which we have been able to obtain applications.

2. For our purposes here it is simplest to specify the language $I$ of elementary number theory as follows. The variable symbols are only those of type 0 , namely, $x, y, z, \ldots$, and these are intended to range over the set $\omega$ of natural numbers. We have distinct constant symbols $\bar{n}$ for $n=0,1,2, \ldots ; \mathrm{L}$ contains four basic relation symbols $\mathbf{R}_{i}, 1 \leqslant i \leqslant 4$, of $l_{i}$ arguments, where $l_{1}=l_{2}=2, l_{3}=l_{4}=3$; these correspond, respectively, to the relations $R_{i}$ given by

$$
\begin{gathered}
R_{1}(x, y) \Leftrightarrow x=y, \quad R_{2}(x, y) \Leftrightarrow x^{\prime}=y, \quad R_{3}(x, y, z) \Longleftrightarrow x+y=z, \\
R_{4}(x, y, z) \Leftrightarrow x \cdot y=z .
\end{gathered}
$$

(The following is easily generalized to deal with more basic relations.) The basic logical symbols are $\sim, \vee, \vee$. The other propositional connectives are assumed to be introduced in any one of the usual ways in terms 
of $\sim$ and $\vee$; universal quantification is introduced by $\wedge x \mathfrak{F}=\sim \vee x \sim F$ The symbols $ᄀ, \&, \Rightarrow, \Longleftrightarrow, \mathbb{H}, \mathrm{F}$ are reserved for abbreviation in informal statements.

Let $\delta$ be fixed, $0<\delta \leqslant \omega$. The auxiliary language $L^{*}=L^{*}\left(\mathbf{S}_{0}, \ldots, \mathbf{S}_{n}, \ldots\right)$ is obtained from $\mathrm{L}$ by simply adjoining unary predicate symbols $\mathbf{S}_{n}$, $0 \leqslant n<\delta$. (In the case that $\delta=1$, we write $\mathbf{S}$ intead of $\mathbf{S}_{0}$.) We write $\mathrm{t} \epsilon \mathbf{S}_{n}$, where $\mathrm{t}$ is a variable or individual constant, instead of $\mathbf{S}_{n}(\mathrm{t})$, and we write $t \dot{\epsilon} \mathbf{S}_{n}$ instead of $\sim\left(\mathrm{t} \epsilon \mathbf{S}_{n}\right)$. By a sentence of $L^{*}$ we mean a formula with no free variables. We shall say that $\mathfrak{F}$ is an arithmetical sentence if it is a sentence of $\mathrm{L}$. By a basic sentence of $\mathrm{L}^{*}$ we mean one of either of the forms $\bar{k} \in \mathbf{S}_{n}$ or $\bar{k} \xi \mathbf{S}_{n}$.

A set $X$ of basic sentences is said to be consistent if there are no $k, n$ with both $\left(\bar{k} \in \mathbf{S}_{n}\right)$ and $\left(\bar{k} \dot{\xi} \mathbf{S}_{n}\right)$ in $X$. By a finite set of conditions we mean a finite consistent set of basic sentences. Throughout the following we use the letters $Q, Q^{\prime}, Q^{\prime \prime}$ (with or without subscripts) to range over finite sets of conditions. A sequence $Q_{0}, \ldots, Q_{m}, \ldots$ of finite sets of conditions is said to be complete if:

(i) $\cup Q_{m}[m<\omega]$ is consistent,

(ii) for any $k, n$ with $0 \leqslant n<\delta$ there exists an $m$ such that $\left(\vec{k} \in \mathbf{S}_{n}\right)$ or $\left(\bar{k} \in \mathbf{S}_{n}\right)$ belongs to $Q_{m}$.

Any complete sequence determines a sequence of sets $S_{0}, \ldots, S_{n}, \ldots$ $(0 \leqslant n<\delta)$ by means of:

(iii) $k \in S_{n} \Longleftrightarrow(\mathbb{H} m)\left[\left(\bar{k}_{\in} \mathbf{S}_{n}\right)\right.$ belongs to $\left.Q_{m}\right]$.

We call this the associated sequence of sets for the given $Q_{m}$ 's. Conversely, given any sequence of sets $S_{0}, \ldots, S_{i}, \ldots(0 \leqslant i<\delta)$, we form its diagram, in symbols, $\operatorname{Diag}\left(S_{0}, \ldots, S_{i}, \ldots\right)$ by:

(iv) $\left(\bar{k} \in \mathbf{S}_{n}\right)$ or $\left(\bar{k} \in \mathbf{S}_{n}\right)$ is in $\operatorname{Diag}\left(S_{0}, \ldots, S_{i}, \ldots\right)$ for each $k, n$ with $0 \leqslant n<\delta$, and

(v) $\left(\bar{k}_{i} \in \mathbf{S}_{n}\right)$ is in $\operatorname{Diag}\left(S_{0}, \ldots, S_{i}, \ldots\right) \Leftrightarrow k \in S_{n}$

Then the collection of all finite subsets of $\operatorname{Diag}\left(S_{0}, \ldots, S_{n}, \ldots\right)$ can be arranged in a complete sequence of conditions whose associated sequence of sets is $S_{0}, \ldots, S_{n}, \ldots$

We now give the definition of forcing for sentences of $L^{*}$, in the inductive form suggested to us by Scott. This is very close to the inductive definition of number-theoretical truth. In fact, the only difference is in the treatment of atomic formulas $\left(\bar{k} \in \mathbf{S}_{n}\right)$ and of negation, as needed to express precisely the intuitive formulation of $\S 1$.

2.1. Definimion. The relation, $Q$ forces $\mathfrak{F}$, in symbots $Q \Vdash \mathfrak{F}$, is defined inductively for arbitrary finite sets of conditions $Q$ and sentences $\&$ of $L^{*}$, as follows: (i) $Q \Vdash\left(\bar{k} \in \mathbf{S}_{n}\right) \Longleftrightarrow\left(\bar{k} \in \mathbf{S}_{n}\right)$ is in $Q$;

(ii) $Q \| \mathbf{R}_{i}\left(\bar{k}_{1}, \ldots, \bar{k}_{i_{i}}\right) \Leftrightarrow R_{i}\left(k_{1}, \ldots, k_{i_{i}}\right)$, for $i=1, \ldots, 4$;

(iii) $Q \Vdash \mathfrak{F} \vee \mathfrak{F} \Leftrightarrow Q \Vdash \mathfrak{F}$ or $Q \Vdash \mathfrak{F}$;

(iv) $Q \Vdash \sim \widetilde{F} \Leftrightarrow \neg\left(\right.$ स $\left.Q^{\prime}\right)\left(Q^{\prime} \supseteq Q \& Q^{\prime} \| \widetilde{F}\right)$;

(v) $Q \|-\bigvee \times \widetilde{F}(\mathrm{x}) \Longleftrightarrow($ 县 $k) Q \| \widetilde{F}(\bar{k})$.

2.2. Definition. A sequence $Q_{0}, \ldots, Q_{m}, \ldots$ is said to be generic $i f$

(i) $\left\lfloor! Q_{m}[m<\omega]\right.$ is consistent, and

(ii) for any sentence $\mathbb{F}$ of $\mathrm{L}^{*}$ there exists an $m$ such that $Q_{m} \| \mathfrak{F}$ or $Q_{m} \| \sim \widetilde{F}$.

A sequence $S_{0}, \ldots, S_{n}, \ldots(0 \leqslant n<\delta)$ of sets is said to be generic if it is the associated sequence of a generic sequence $Q_{0}, \ldots, Q_{m}, \ldots$

Clearly, any generic sequence $Q_{0}, \ldots, Q_{m}, \ldots$ is complete. Also $S_{0}, \ldots, S_{n}, \ldots$ is generic if and only if any enumeration of the finite subsets of $\operatorname{Diag}\left(S_{0}, \ldots, S_{n}, \ldots\right)$ is generic-hence, if and only if for any sentence $\widetilde{F}$ of $\mathrm{L}^{*}$

$$
(\mathbb{H} Q)\left[Q \subseteq \operatorname{Diag}\left(S_{0}, \ldots, S_{n}, \ldots\right) \&(Q\|\widetilde{F} \vee Q\| \sim F)\right] .
$$

For any $S_{0}, \ldots, S_{n}, \ldots \quad(0 \leqslant n<\delta)$, let $\mathcal{M}^{*}=\mathcal{H}^{*}\left(S_{0}, \ldots, S_{n}, \ldots\right)$ be the structure $\left\langle\omega, R_{1}, R_{2}, R_{3}, R_{4}, S_{0}, \ldots, S_{n}, \ldots\right\rangle$. As usual, $\vDash \mathcal{N} * \widetilde{f}$ means that (the sentence) $\mathbb{F}$ is true in $\mathcal{K}^{*}$. If $\mathfrak{F}$ is a sentence of L we say that $\mathfrak{F}$ is true in the natural numbers if it is true in $\mathcal{H}^{*}$, equivalently if it is true in the structure $\left\langle\omega, R_{1}, R_{2}, R_{3}, R_{4}\right\rangle$.

Following Cohen [2] or [3], we can easily derive the next results 2.3-2.4 from the basic definitions. In particular, the important property 2.4 (ii) is immediate from 2.1 (iv).

2.3. THEOREM.

(i) If $\widetilde{\Im}$ is an arithmetical sentence then $Q \| \widetilde{F}$ if and only if $\widetilde{F}$ is true in the natural numbers.

(ii) $Q \Vdash\left(\bar{k} \dot{\xi} \mathbf{S}_{n}\right) \Longleftrightarrow\left(\bar{k} \dot{\epsilon} \mathbf{S}_{n}\right)$ is in $Q$.

(iii) $Q \Vdash \wedge \mathrm{x} \mathscr{F}(\mathrm{x}) \Longleftrightarrow(\nabla K)\left(\nabla Q^{\prime}\right)\left\{Q^{\prime} \supseteq \underline{Q} Q \Rightarrow\left(\mathbb{B} Q^{\prime \prime}\right)\left[Q^{\prime \prime} \supseteq Q^{\prime} \& Q^{\prime \prime} \| \mathfrak{F}(\bar{k})\right]\right\}$.

2.4. THEOREM.

(i) $Q \| \mathscr{F} \& Q \underline{C} Q^{\prime} \Rightarrow Q^{\prime} \mid-\widetilde{F}$.

(ii) $(\nabla Q)(\nabla \mathscr{F})\left\{\widetilde{F}\right.$ a sentence of $\left.\mathrm{L}^{*} \Rightarrow\left(\mathbb{H} Q^{\prime}\right)\left[Q^{\prime} \geq Q \&\left(Q^{\prime} \| \mathfrak{F} \vee Q^{\prime} \mid-\sim \mathfrak{F}\right)\right]\right\}$. This leads directly to the following result.

2.5. THEOREM. Given any $\delta, 0<\delta \leqslant \omega$, there exists a generic sequence $S_{0}, \ldots, S_{n}, \ldots(0 \leqslant n<\delta)$.

Proof. We shall construct a generic sequence of conditions $Q_{0}, \ldots, Q_{m}, \ldots$ By 2.4 (ii) there exists a function $g(Q, \widetilde{F})$ such that for each $Q$ and each sentence $\mathfrak{F}$ of $L^{*}$, 
(1) $g(Q, \mathbb{F})$ is a finite set of conditions and $Q \subset g(Q, \mathfrak{F})$, and

(2) $g(Q, \mathfrak{F}) \Vdash \mathfrak{F}$ or $g(Q, \mathfrak{F}) \mid \sim \sim \mathfrak{F}$.

Let $\widetilde{\mho}_{0}, \ldots, \widetilde{\mho}_{m}, \ldots$ be an enumeration of all sentences of $L^{*}$. Then define

(3) $Q_{0}=\emptyset$ and $Q_{m+1}=g\left(Q_{m}, \widetilde{F}_{m}\right)$ for any $m$.

This gives the desired result.

The next is the basic reduction theorem due to Cohen, referred to in $\S 1$ (cf. [3], Part I, Lemma כ).

2.6. THEOREM. Suppose that $S_{0}, \ldots, S_{n}, \ldots$ is a generic sequence and that $\aleph^{*}=M^{*}\left(S_{0}, \ldots, S_{n}, \ldots\right)$. Let $D=\operatorname{Diag}\left(S_{0}, \ldots, S_{n}, \ldots\right)$. Then for any sentence $\mathfrak{F}$ of $\mathrm{L}^{*}$;

$$
\vDash \nVdash * \widetilde{F} \Leftrightarrow(\mathbb{H} Q)[Q \subseteq D \& Q \| \widetilde{F}] .
$$

Proof. This is proved by induction on $\mathfrak{F}$. It is seen for atomic $\widetilde{F}$ by 2.1 (i), (ii). The passage to $V$ and $V$ is ensured by 2.1 (iii), ( $v$ ). Suppose $=\mathcal{H}_{*} \sim \mathfrak{F}$. Then 갸 $=\mathcal{H}_{*} \approx \mathfrak{F}$ so that, by induction hypothesis, for each $Q \subseteq D$ we have $\neg(Q \| \mathfrak{F})$. But then by the definition 2.2 of generic sequence, there must exist $Q \subseteq D$ with $Q \| \sim F$. Conversely, suppose $Q \subseteq D$ and $Q \| \sim \widetilde{F}$. For any $Q^{\prime} C^{\prime} D, Q \cup Q^{\prime}$ is consistent and $Q \subseteq Q \cup Q^{\prime}$. Hence by 2.4 (i), $\left(Q \cup Q^{\prime}\right) \| \sim \widetilde{F}$. But then by 2.1 (iv) and 2.4 (i), $\neg\left(Q^{\prime} \mid-\mathscr{F}\right)$. Thus $\mathfrak{F}$ is not true in $\mathbb{K}^{*}$, by induction hypothesis, and $\models \mathbb{N}^{*} \sim \widetilde{F}$.

Clearly, the converse theorem is also true, i.e. if $S_{0}, \ldots, S_{n}, \ldots$ is any sequence for which the conctusion of 2.6 holds then it is a generic sequence. (This lends added justification for the present use of the term "generic".)

In proving the independence of the axiom of choice in his paper [2], Cohen introduced certain symmetries into the model he constructed by considering permutations $\pi$ of $\omega$. Corresponding to any such permutation there is a permutation of the basic symbols given by sending $\mathbf{S}_{n}$ into $\mathbf{S}_{\pi(n)}$. This induces a transformation of finite sets of conditions $Q$ and of sentences $\widetilde{F}$, denoted by $\pi(Q)$ and $\pi(\mathfrak{F})$, respectively. Namely, $\pi(Q)$ consists of all sentences $\bar{k} \in \mathbf{S}_{x(n)}$ (resp., $\bar{k} \dot{\epsilon} \mathbf{S}_{x(n)}$ ) such that $\bar{k} \in \mathbf{S}_{n}$ (resp., $\bar{k} \in \mathbf{S}_{n}$ ) belongs to $Q$, and $\pi(\mathfrak{F})$ is obtained from $\mathscr{F}$ by replacing each occurence of the form $\left(t \in \mathbf{S}_{n}\right)$ in $\tilde{F}$ by $t \in \mathbf{S}_{\pi(n)}$. Then

$$
Q-\mathfrak{F} \Leftrightarrow \pi(Q) \|-\pi(\widetilde{\mho}) \text {. }
$$
The desired symmetry is obtained for those $Q$ and $\pi$ such that
$\pi(Q)=Q$.

In this paper we make use of a different kind of transformation which proves useful in a variety of situations. Let $\tau$ be a function defined for any $k, n$ with $0 \leqslant n<\delta$, but taking only the values 0 and 1 . Given ang sequence $S_{0}, \ldots, S_{n}, \ldots(0 \leqslant n<\delta)$, consider the sequence
$S_{0}^{(\tau)}, \ldots, S_{n}^{(\tau)}, \ldots$ obtained from it by taking

$$
k \in S_{n}^{(\tau)} \Leftrightarrow\left\{\begin{array}{lll}
k \in S_{n} & \text { if } & \tau(k, n)=1, \\
k \in S_{n} & \text { if } & \tau(k, n)=0 .
\end{array}\right.
$$

Thus, for example, if for given $n, \tau(k, n)=1$ for all but finitely many values of $k$, then $S_{n}^{(t)}$ and $S_{n}$ agree except for finitely many elements. On the other hand, if $\tau(k, n)=0$ for all but finitely many $k$, then $S_{n}^{(\tau)}$ agrees with $\omega-S_{n}$ except for finitely many elements. It is clear how to associate with an arbitrary such $\tau$ an associated transformation of conditions $Q$ into $\tau(Q)$. However, in order to define the associated transformation of sentences, we need, in general, a formal definition of the function $\tau$ in $L^{*}$. For the main transform theorem we obtain in 2.8 below, it is further necessary that this definition be "absolute", i.e. be given by an arithmetical formula (formula of $L$ ). Let $\mathfrak{I}(x, y, z)$ be such a formula with the free variables $\mathbb{x}, \bar{y}, \mathbf{z}$. We say $\mathfrak{I}$ defines $\tau$ if for any $k, i, n(0 \leqslant n<\delta)$ we have

$$
\tau(k, n)=i \Leftrightarrow \mathfrak{I}(\bar{k}, \bar{n}, \bar{i}) \text { is true in the natural numbers. }
$$

We assume throughout the following that $\tau$ is arithmetically definable by $\mathfrak{I}$ in this sense.

2.7. DeFrnimion. (i) Fror any $Q, \tau(Q)$ is taken to consist of all sentences of the form $\left(\bar{k} \in \mathbf{S}_{n}\right)$ such that $\left[\left(\bar{k} \in \mathbf{S}_{n}\right)\right.$ is in $Q$ and $\tau(k, n)=1$ or $\left(\bar{k} \dot{\phi} \mathbf{S}_{n}\right)$ is in $Q$ and $\tau(k, n)=0]$, together with all sentences of the form $\left(\bar{k} \dot{\epsilon} \mathbf{S}_{n}\right)$ such that $\left[\left(\bar{k} \epsilon \mathbf{S}_{n}\right)\right.$ is in $Q$ and $\tau(k, n)=1$ or $\left(\bar{k} \in \mathbf{S}_{n}\right)$ is in $Q$ and $\tau(k, n)$ $=0]$.

(ii) For any formula $\mathfrak{F}$ of $\mathrm{L}^{*}, \tau(\mathfrak{F})$ is obtained from $\mathfrak{F}$ by replacing each occurrence of an atomic formula $\left(\mathrm{s} \in \mathbf{S}_{n}\right)$ in $\mathfrak{F}$ (s variable or constant, by $\left(\left[\mathfrak{T}(\mathrm{s}, \bar{n}, \overline{1}) \wedge \mathrm{s} \in S_{n}\right] \vee\left[\mathcal{G}(\mathrm{s}, \bar{n}, \overline{0}) \wedge \mathrm{s} \dot{\epsilon} \mathbf{S}_{n}\right]\right)$.

Note that if $Q \subseteq Q^{\prime}$ then $\tau(Q) \subseteq \tau\left(Q^{\prime}\right)$ and that $\tau(\tau(Q))=Q$ for any $Q$.

3.8. THEOREM.

$$
Q \mid-\mathfrak{F} \Leftrightarrow \tau(Q)-\tau(\mathfrak{F}) .
$$

Proof. This statement, when preceded by ' $\nabla Q$ ' is proved by induction on $\mathfrak{F}$. For atomic $\mathfrak{F}$ we make use of $\mathbf{2 . 3}$ (i) and the fact that $\mathfrak{I}$ is an arithmetical definition of $\tau$. Since $\tau$ preserves $V$ and $V$, the inductive passage for these is immediate. $\tau$ also preserves $\sim$. Hence it is sufficient, assuming the inductive hypothesis, to show for any $Q$ that

$$
\left(\mathbb{H} Q^{\prime}\right)\left(Q^{\prime} \supseteq Q \& Q^{\prime} \mid-\mathfrak{F}\right) \Leftrightarrow\left(\mathbb{H} Q^{\prime \prime}\right)\left(Q^{\prime \prime} \subseteq \tau(Q) \& Q^{\prime \prime} \mid-\tau(\mathfrak{F})\right) .
$$

Indeed, we get $\Rightarrow$ from the fact that $Q^{\prime} \subseteq Q$ implies $\tau\left(Q^{\prime}\right) \supseteq \tau(Q)$. For the converse, if $Q^{\prime \prime} \supseteq \tau(Q)$ and we put $Q^{\prime}=\tau\left(Q^{\prime \prime}\right)$, we have $\tau\left(Q^{\prime}\right)=\tau\left(\tau\left(Q^{\prime \prime}\right)\right)$. 
$=Q^{\prime \prime}$, and $Q^{\prime} \supseteq Q$. Thus the induction hypothesis applied to $Q^{\prime}$ gives the desired result.

This concludes that part of the theory of forcing and generic sets which we shall see carries over intact to other languages. We now turn to consider results which are more specific to the language of number theory. In particular, we want now to obtain a more constructive description of how a generic sequence $S_{0}, \ldots, S_{n}, \ldots$ can be defined. For this purpose we make use of the theory of hyperarithmetical sets, with which we now assume some familiarity $\left({ }^{2}\right)$. A set $B$ of natural numbers is said to be a $\prod_{1}^{1}$ set or predicate if for some arithmetical predicate $A(x, X)$, where $X$ ranges over subsets of $\omega$, we have for every $k$,

$$
k \in B \Leftrightarrow(\nabla X) A(k, X) .
$$

We say $B$ is a $\sum_{1}^{1}$ predicate if $\omega-B$ is $\prod_{1}^{1} . B$ is said to be hyperarithmetical if it is both $\prod_{1}^{1}$ and $\sum_{1}^{1}$. These notions are extended in the obvious way to relations, e.g. by identifying $n$-tuples $\left\langle k_{1}, \ldots, k_{n}\right\rangle$ of natural numbers with natural numbers $p_{1}^{k_{1}} \ldots p_{n}^{k_{n}}$; we do so throughout the following. A function is said to be hyperarithmetical if its graph is. We also apply these notions to predicates or functions of expressions and of finite sequences and sets of expressions in a formalized language, by means of any one of the standard effective one-to-one correspondences between expressions and numbers (Gödel-numbering). Finally, we will also make use of relativized versions of all these notions, such as that of being hyperarithmetic(al) in (given sets).

2.9. THEOREM. The relation, $Q \Vdash \mathfrak{F}$, is a $\prod_{1}^{1}$ predicate of $Q$ and $\mathfrak{F}$; in fact, it is hyperarithmetical.

Proof. The proof is very similar to that for establishing that the set of true arithmetical sentences is $\Pi_{1}^{1}$ and thence hyperarithmetical. However, for purposes of reference in $\S 3$, we briefly describe how it is carried out. One first defines a set $K$ of triples $\langle Q, \widetilde{F}, i\rangle$, where $Q$ is a finite set of conditions, $₹$ is a sentence of $\mathrm{L}^{*}, i=0$ or 1 . This shall have the property that for any $Q$ and $\mathrm{F}$,

$$
Q \Vdash \mathfrak{F} \Leftrightarrow\langle Q, \mathfrak{F}, 1\rangle \epsilon K \Leftrightarrow\langle Q, \widetilde{F}, 0\rangle \xi K .
$$

Namely, $K$ is described as the intersection of all sets $X$ satisfying certain closure conditions, corresponding to 2.1 (i)-(v). For example the closure conditions corresponding to $2.1(\mathrm{v})$ are:

(2) if for some $k,\langle Q, \mathfrak{F}(\bar{k}), 1\rangle \epsilon X$ then $\langle Q, \bigvee \times \mathfrak{F}(\mathrm{x}), 1\rangle \epsilon X$;

$$
\text { if for all } k,\langle Q, \mathfrak{F}(\bar{k}), 0\rangle \in \bar{X} \text { then }\langle Q, \bigvee \times \mathbb{F}(\mathrm{x}), 0\rangle \in X \text {. }
$$

(2) Most of the basic notions and results of this field can be found in Kleene [9]
Spector [16].
The closure conditions corresponding to 2.1 (iv) are:

(3) if for some $Q^{\prime} \geq Q,\left\langle Q^{\prime}, \mathfrak{F}, 1\right\rangle \in X$ then $\langle Q, \sim \mathscr{F}, 0\rangle \in X$;

if for all $Q^{\prime} \geq Q,\left\langle Q^{\prime}, \widetilde{F}, 0\right\rangle \in X$ then $\langle Q, \sim \mathscr{F}, 1\rangle \in X$.

These closure conditions are arithmetical in $X$. Hence the set $K$ is seen to be $\eta_{1}^{1}$. Then one shows that $K$ satisfies the same closure conditions. Finally, we prove (1) by induction on $\widetilde{F}$, using the pairing of the closure conditions on $K$ as in (2) and (3). By the second equivalence in (1), the forcing relation is hyperarithmetical.

2.10. THEOREM. There exists a hyperarithmetical set $S$ such that the sequence of sets $S_{n}$, determined by $S_{n}=\{k:\langle k, n\rangle \in S\}$ for $0 \leqslant n<\delta$, is generic.

Proof. We make use of the following theorem proved by Kreisel ([12], p. 307):

(1) If $P(x, y)$ is a $\prod_{1}^{1}$ predicate such that $(\nabla x)(\mathbb{H} y) P(x, y)$ then there exists a hyperarithmetical function $g$ such that $(\nabla x) P(x, g(x))$.

(In fact, Kreisel shows explicitly how to define $g$ given a definition of $P$.) Now by 2.4 (ii) we have

(2) $(\nabla Q, \widetilde{F})\left(\mathbb{H} Q^{\prime}\right)\left[\mathscr{F}\right.$ a sentence of $\left.\mathrm{L}^{*} \Rightarrow Q^{\prime} \beth Q \&\left(Q^{\prime}\left\|\mathfrak{F} \vee Q^{\prime}\right\| \sim \widetilde{F}\right)\right]$.

Hence by 2.9 and Kreisel's theorem (1), we can find hyperarithmetical $g$ satisfying the conditions (1) and (2) of the proof of 2.5. Then the sequence $Q_{0}, \ldots, Q_{m}, \ldots$ defined from $g$ in (3) of that proof is also hyperarithmetical. Finally, we determine the required $S$ by, $\langle k, n\rangle \in S \Leftrightarrow(\mathbb{H} m)$ $\left[\left(\bar{k} \in \mathbf{S}_{n}\right)\right.$ is in $\left.Q_{m}\right]$.

2.11. DefintTion. (i) Suppose given subsets $B, C_{1}, \ldots, C_{i-1}$ of $\omega(i \geqslant 1)$. We say that $B$ is arithmetically dependent on $C_{1}, \ldots, C_{i-1}$ if for some arithmetical predicate $A\left(X, Y_{1}, \ldots, Y_{i-1}\right)$,

$$
B=\text { the unique } X \text { such that } A\left(X, C_{1}, \ldots, C_{i-1}\right) .
$$

(ii) $A$ (finite or infinite) sequence $B_{0}, \ldots, B_{n}, \ldots$ of sets is said to be arithmetically independent if for no $n, m_{1}, \ldots, m_{i-1}$ with $i \geqslant 1$ and $n \neq m_{1}, \ldots, m_{i-1}$, do we have $B_{n}$ arithmetically dependent on $B_{m_{1}}, \ldots, B_{m_{i-1}}$.

Clearly, if a set $B$ is arithmetically definable in terms of sets $C_{1}, \ldots, C_{i-1}$, then it is arithmetically dependent on these sets. In particular, every arithmetical set is arithmetically dependent on the empty set. However, the converse is far from true. For example, it is well known that for each of the sets $H_{e}(e \epsilon O)$ in Kleene's hyperarithmetical hierarchy, we have an arithmetical predicate $A_{e}(X)$ such that

$$
H_{e}=\text { the unique } X \text { such that } A_{e}(X) \text {. }
$$


Every hyperarithmetical set is recursive in some $H_{e}$ for $e \epsilon O$. Kreisel raised the question whether every hyperarithmetical set is the unique solution of an arithmetical predicate. Despite the preceding result concerning the $H_{e}$ 's, the answer is shown to be negative by part (iii) of the next theorem.

2.12. THEOREM. (i) Any generic sequence of sets $s_{0}, \ldots, S_{n}, \ldots(0 \leqslant n$ $<\delta)$ is arithmetically independent.

(ii) There exist hyperarithmetical, arithmetically independent sets $S_{0}, \ldots, S_{n}, \ldots(0 \leqslant n<\omega)$.

(iii) In particular, there exist hyperarithmetical sets $S$ which are not a unique solution $X$ of any arithmetical predicate $A(X)$.

Proof. (ii) and (iii) are immediate corollaries of (i) by 2.10. To prove (i), let $S_{0}, \ldots, S_{n}, \ldots$ be any generic sequence of sets. We show, as a typical case, that $S_{0}$ is not arithmetically dependent on $S_{1}, \ldots, S_{i-1}$, where $i \geqslant 1$. Note that we can associate with any arithmetical predicate $A\left(Y_{0}, Y_{1}, \ldots, Y_{i-1}\right)$ a sentence $\mathfrak{F}$ of $L^{*}$ such that $\mathfrak{F}$ contains only the constants $\mathbf{S}_{0}, \ldots, \mathbf{S}_{i-1}$ and such that $A\left(S_{0}, S_{1}, \ldots, S_{i-1}^{\prime \prime}\right)$ holds if and only if $\widetilde{\mho}$ is true in $\mathfrak{H}^{*}$. We write $\mathfrak{F}=\mathfrak{F}\left(\mathbf{S}_{0}, \mathbf{S}_{1}, \ldots, \mathbf{S}_{i-1}\right)$. Now suppose the predicate $A$ is such that $A\left(S_{0}, S_{1}, \ldots, S_{i-1}^{\prime}\right)$. Taking the corresponding sentence $\mathfrak{F}\left(\mathbf{S}_{0}, \mathbf{S}_{1}, \ldots, \mathbf{S}_{i-1}\right)$ we thus have $/{ }_{\mathcal{M}} * \mathfrak{F}\left(\mathbf{S}_{0}, \mathbf{S}_{1}, \ldots, \mathbf{S}_{i-1}\right)$. Hence by the basic reduction theorem 2.6, we can find $Q$ such that

$$
Q \subseteq \operatorname{Diag}\left(S_{0}, \ldots, S_{n}, \ldots\right) \quad \text { and } \quad Q \| \mathfrak{F}\left(\mathbf{S}_{0}, \mathbf{S}_{1}, \ldots, \mathbf{S}_{i-1}\right)
$$

Consider the least $k_{0}$ such that neither $\left(\bar{k}_{0} \in \mathbf{S}_{0}\right)$ nor $\left(\bar{k}_{0} \epsilon \mathbf{S}_{0}\right)$ belongs to $Q$. Then define

$$
\tau(k, n)=\left\{\begin{array}{lll}
1 & \text { if } \quad n \neq 0 \text { or } n=0 \& k \neq k_{0}, \\
0 & \text { if } \quad n=0 \text { and } k=k_{0} .
\end{array}\right.
$$

Thus the sequence of sets $S_{0}^{(\tau)}, \ldots, S_{n}^{(\tau)}, \ldots$ obtained from $S_{0}, \ldots, S_{n}, \ldots$ by the transform $\tau$ is such that $S_{0}^{(\tau)}$ differs from $S_{0}$ exactly at the one point $k_{0}$, while $S_{n}^{(\tau)}=S_{n}$ for $n>0$. Since $\tau(Q)=Q$ by choice of $k_{0}$,
we have

$$
Q \Vdash \tau\left(\mathfrak{F}\left(\mathbf{S}_{0}, \mathbf{S}_{1}, \ldots, \mathbf{S}_{i-1}\right)\right) .
$$

by 2.8 , and hence by 2.6

$$
1=\mathcal{H} * \tau\left(\widetilde{\mho}\left(\mathbf{S}_{0}, \mathbf{S}_{1}, \ldots, \mathbf{S}_{i-1}\right)\right) .
$$

We then see from 2.7 (ii) that (4) formally expresses that $A\left(S_{0}^{(\tau)}, S_{1}, \ldots, S_{i-1}\right)$. Hence $S_{0}$ is not a unique solution $X$ of $A\left(X, S_{1}, \ldots, S_{i-1}\right)$.

We have not been able to find any direct, e.g. diagonal, argument which would establish even (iii).
Consider the case $\delta=1$, so that we are dealing with a single predicate symbol $\mathbf{S}$ when forming $\mathrm{L}^{*}$, and we have only a single generic set $S$ relative to this language. Let $S_{n}=\{k:\langle k, n\rangle \in S\}$. Then the sequence $S_{0}, S_{1}, \ldots, S_{n}, \ldots$ has many of the properties of a generic sequence for $\delta=\omega\left(^{3}\right)$. In particular, by an argument quite similar to the one just given, we can obtain the following theorem.

2.13. THEOREM. Suppose that $\delta=1$ and that $S$ is generic for the language $\mathrm{L}^{*}=\mathrm{L}^{*}(\mathbf{S})$. Let $S_{n}=\{k:\langle k, n\rangle \in S\}$. Then the sequence of sets $S_{0}, \ldots, S_{n}, \ldots$ is arithmetically independent.

3. We now turn to a discussion of the notions of forcing and generic sets with respect to certain 2 nd order languages. Thus, in the terms of $\S 1, \beta_{0}=2$; for the moment, let $\alpha_{0}$ be any fixed denumerable limit ordinal. The basic language $L$ is that of 1st order number theory (§2) extended by the introduction of 2 nd order variables $X, Y, Z, \ldots$, intended to range over subsets of $\omega$, and by the binary relation symbol $\epsilon$. The auxiliary language $\mathrm{L}^{*}=\mathrm{L}^{*}\left(\mathbf{S}_{0}, \ldots, \mathbf{S}_{n}, \ldots\right), 0 \leqslant n<\delta$, is an extension of $\mathrm{L}$ by the (now) 2 nd order constant symbols $\mathbf{S}_{0}, \ldots, \mathbf{S}_{n}, \ldots$ In addition, $\mathrm{L}^{*}$ contains for each $a<\alpha_{0}$ a stock of distinct 2 nd order variable symbols $\mathrm{X}^{\alpha}, \mathrm{Y}^{\alpha}, \mathrm{Z}^{\alpha}, \ldots$ of rank $\alpha$ (not to be confused with the set-theoretical notion of rank). The intention is that the variables of rank $a$ shall range over sets defined by formulas involving only 2nd order quantifications with variables of ranks less than $\alpha$. We say that $\mathrm{L}^{*}$ is a 2 nd order ramified language of rank $a_{0}$. Formulas of $\mathrm{L}^{*}$ may involve both ranked and unranked second order variables.

To be more precise about the foregoing, we say a formula $\widetilde{F}$ is (completely) ranked if each 2 nd order variable in $\mathfrak{F}$ has some rank $\alpha<\alpha_{0}$. If $\widetilde{F}$ is ranked, we denote by $\varrho(\mathfrak{F})$ the maximum of all $\alpha$ such that a variable $X^{\alpha}$ is free in $\widetilde{F}$ and of all $\beta+1$ such that a variable $X^{\beta}$ is bound in $\widetilde{F} ; \varrho(\widetilde{F})=0$ if there are no such variables. We say that an arbitrary formula $\widetilde{F}$ is arithmetical (in its free variables) if it contains no 2 nd order quantifiers. For $\widetilde{F}$ ranked this is equivalent to $\varrho(\mathfrak{F})=0$.

Suppose given any sets $S_{0}, \ldots, S_{n}, \ldots, 0 \leqslant n<\delta$. We define structures $\mathcal{H}_{a}^{*}=\mathcal{M}_{a}^{*}\left(S_{0}, \ldots, S_{n}, \ldots\right)=\left\langle M_{a}^{*}, \epsilon\right\rangle$ and the notion of truth in $\left\langle\mathcal{K}_{\beta}^{*}\right\rangle_{\beta<a}$ recursively, for $\alpha \leqslant \alpha_{0}$, by the following conditions:

(i) A ranked sentence $\mathfrak{F}$ with $\varrho(\mathfrak{F}) \leqslant \alpha$ is said to be true in $\left\langle\mathcal{M}_{\beta}^{*}\right\rangle_{\beta<\alpha}$ if it is true when the 1 -st order variables are interpreted to range over $\omega$ and each $2-n d$ order variable of rank $\beta(<\alpha)$ is interpreted to range over $M_{\beta}^{*}$.

(3) In fact, and as is easily seen, it is a generic sequence for $\delta=\omega$ in the case of number theory. However, we do not know if this extends to the languages $\mathrm{L}^{*}$ considered below. In any case, 2.13 does extend to these languages. 
(ii) For each ranked formula $\mathfrak{G}(\mathrm{x})$ with just $\mathrm{x}$ free and $\varrho(\mathfrak{5}(\mathrm{x})) \leqslant \alpha$, take $B_{5}=\left\{n: \mathscr{G}_{(\bar{n})}\right.$ is true in $\left.\left\langle M_{\beta}^{*}\right\rangle_{\beta<a}\right\}$. Then $M_{a}^{*}$ consists of all such sets $B_{\mathfrak{G}}$, and only of such sets.

It is seen that $\mathcal{H}_{0}^{*}=\mathcal{M}_{0}^{*}\left(S_{0}, \ldots, S_{n}, \ldots\right)$ consists just of the sets $B$ arithmetically definable in $S_{0}, \ldots, S_{n}, \ldots$ Furthermore, $M_{\beta}^{*} \subseteq M_{\alpha}^{*}$ for $\beta<\alpha$. We take $\mathscr{H}^{*}=\mathscr{H}_{a_{0}}^{*}$.

To extend the definition of forcing to $\mathrm{L}^{*}$, we need a means of describing substitution for 2nd order variables by particular formal definitions. Given $\widetilde{F}(U)$, where $U$ may be ranked or unranked, and $\mathfrak{G}(x)$ containing a distinguished 1st order variable $x$, we write $\mathfrak{F}(\hat{x}(\mathfrak{F}(x))$ for the result of replacing each occurrence of an atomic formula $(t \in U)$ in $\mathfrak{F}$ by $\mathfrak{b}(\mathrm{t})$.

3.1. Defrnition. The relation, $Q \Vdash \mathfrak{F}$, for $\mathfrak{F}$ a ranked sentence of $\mathrm{L}^{*}$ is defined inductively as follows. For atomic sentences, disjunction, negation, and numerical existential quantification, we use exactly the same conditions as in 2.1 (i)-(v). In addition, we take:

(vi) $Q \|-V X^{\alpha} \mathfrak{F}\left(X^{\alpha}\right) \Longleftrightarrow$ for some ranked formula $\mathfrak{G}(\mathrm{x})$ with just $\mathrm{x}$ free and $\varrho(\mathfrak{G}(\mathrm{x})) \leqslant \alpha, Q \|-\mathfrak{F}(\hat{\mathrm{x}}(\mathfrak{5}(\mathrm{x}))$.

The relation, $Q \| \widetilde{F}$, is then extended to arbitrary sentences $\mathfrak{F}$ of $\mathrm{L}^{*}$ by

(vii) $Q-\vee \mathrm{X} F(\mathrm{X}) \Longleftrightarrow(\mathbb{H} \alpha)\left[\alpha<\alpha_{0} \& Q \|-\vee \mathrm{X}^{\alpha} \mathfrak{F}\left(\mathrm{X}^{\alpha}\right)\right]$.

That these inductive conditions well-determine the relation $\|$ can be seen by using an argument due to Schütte ([14], p. 250).

If we now read the relation of forcing in this new sense the definition 2.2 gives us a corresponding definition of generic sequences of conditions $Q_{0}, \ldots, Q_{m}, \ldots$ and of sets $S_{0}, \ldots, S_{n}, \ldots$ Definition 2.7 is carried over without change. Then we easily obtain the following.

3.2. THEOREM. All the results 2.3-2.6 and 2.8 continue to hold for the new definition of $t-$ and generic sequences.

Proof. The only really new point to be considered is in the proof of what corresponds here to 2.8. Note that if $\mathfrak{F}$ is ranked then so is $\tau(\mathfrak{F})$ and $\varrho(\tau(\mathfrak{F}))=\varrho(\mathfrak{F})$. Further, $\tau\left(\bigvee X^{\alpha} \mathfrak{F}\left(X^{\alpha}\right)\right)=\bigvee X^{\alpha} \tau\left(\mathfrak{F}\left(X^{\alpha}\right)\right)=\bigvee X^{a} \mathfrak{F}^{\tau}\left(X^{a}\right)$ and $\tau\left(\widetilde{\mho}(\hat{x}(\mathfrak{\zeta}(\mathrm{x})))=\widetilde{J}^{x}(\hat{\mathrm{x}} \tau(\mathfrak{G}(\mathrm{x})))\right.$.

We shall refer to these extended results as $3.3,3.4,3.5,3.6$ and 3.8 , respectively. To obtain further results corresponding to the other theorems of $\S 2$, we need to consider more closely the choice of $\alpha_{0}$ and the

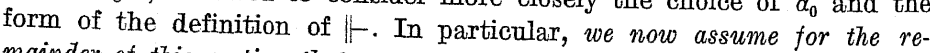
mainder of this section that $\alpha_{0}=\omega_{1}=$ least non-recursive ordinal.

Let $O$ be the set of recursive ordinal notations in the sense of Kleene [9], with each $\alpha<\omega_{1}$ being $\alpha=|a|$ for some $a \in O$. By. Gandy [6] or our paper with Spector [4], we can find a $\prod_{1}^{1}$ subset $O_{1}$ of $O$ which contains a unique notation $a=|\alpha|$ for each $\alpha<\omega_{1}$. Furthermore, $O_{1}$ can be chosen to be well-ordered by a certain recursively enumerable relation $\zeta$ which contains $O_{1}$ in its field. The language $\mathrm{L}^{*}$ is isomorphic to one in which we use variables $\mathrm{X}^{a}, \mathrm{Y}^{a}, \ldots$ for each $a \in O_{1}$. (The rank function $\varrho$ now takes values in $O_{1}$.) Then the notions and results 3.1-3.8 transfer directly to this new language. Since we can now assign Gödelnumbers to expressions in this isomorphic language, we can undertake to classify the relation $1-$ in the analytic hierarchy. Note first, though, that the set of sentences of $\mathrm{L}^{*}$ is no longer a recursive set, as it is in the 1st order case. It is, however, a $\prod_{1}^{1}$ set; the same holds true for the set of ranked sentences.

3.9. THEOREM. (i) The relation between $Q$ and $\mathfrak{F}$, which holds if and only. if $\mathfrak{F}$ is a ranked sentence of $\mathrm{L}^{*}$ and $Q \| \mathfrak{F}$, is $I \prod_{1}^{1}$.

(ii) The relation between $Q$ and $\mathfrak{F}$, which holds if and only if $\mathfrak{F}$ is an arbitrary sentence of $\mathrm{L}^{*}$ and $Q \Vdash \widetilde{F}$, is hyperarithmetical in 0 .

Proof. This follows the lines of proof of 2.9. For (i), we introduce a set $K$ of triples $\langle Q, \widetilde{\mho}, i\rangle$ for which it will be shown that

(1) $\mathfrak{F}$ a ranked sentence of $\mathrm{L}^{*} \Rightarrow[Q \mid \mathfrak{F} \Longleftrightarrow\langle Q, \mathfrak{F}, 0\rangle \in K \Longleftrightarrow\langle Q, \mathfrak{F}, 1\rangle \in K]$. $K$ is again described as the intersection of all sets $X$ satisfying certain closure conditions, such as those given in the proof of 2.9 , together with the following:

(2) if for some ranked $\mathfrak{G}(\mathrm{x})$ with $\varrho(\mathfrak{G}(\mathrm{x})) \preceq a,\langle Q, \mathfrak{F}(\hat{\mathrm{x}}(\mathfrak{5}(\mathrm{x})), 1\rangle \in X$ then $\left\langle Q, \vee X^{a} \mathfrak{F}\left(X^{a}\right), 1\right\rangle \in X$; if for all ranked $\mathfrak{G}(x)$ with $\varrho(\mathfrak{F}(\mathrm{x})) \preceq a$, $\langle Q, \mathfrak{F}(\hat{\mathrm{x}} \mathfrak{G}(\mathrm{x})), 0\rangle \in K$ then $\left\langle Q, \vee \mathrm{X}^{a} \mathfrak{F}\left(\mathrm{X}^{a}\right), 0\right\rangle \in X$

Because $\zeta$ is recursively enumerable, these are again arithmetical closure conditions, so that $K$ is a $\prod_{1}^{1}$ predicate. This leads us to the conclusion (i). Howerer, because of the hypothesis in (1), we can not conclude in this case that $\|$ - is hyperarithmetical. In the proof of (ii), in light of 3.1 (vii), we make use of closure conditions arithmetical in $O_{1}$ and hence in $O$. Then the general relation of forcing is both $\Pi_{1}^{1}$ and $\sum_{1}^{1}$ in $O$.

By relativizing the argument of 2.10 to $O$, we next obtain the following from 3.9 (ii).

3.10. THEOREM. There exists a set $S$, hyperarithmetical in $O$, such that the sequence of sets $S_{n}$, determined by $S_{n}=\{k:\langle k, n\rangle \in S\}$ for $0 \leqslant n<\delta$, is generic.

We could consider a restriction on the notion of generic sequence, by requiring only that for each ranked sentence $\mathfrak{F}$ there exists $Q \subseteq$ $\subseteq \operatorname{Diag}\left(S_{0}, \ldots, S_{n}, \ldots\right)$ such that $Q \| \mathfrak{F}$ or $Q \Vdash \sim \mathfrak{F}$. It is then easily seen that there exists a set $S$ recursive in $O$, such that the associated sequence of sets $S_{n}=\{k:\langle k, n\rangle \in S\}$ is generic in this restricted sense. 
We now turn to giving an appropriate definition of independence of sets in this context, so as to get a theorem corresponding to 2.12 . We say that a formula $\mathfrak{F}$ of $\mathrm{L}^{*}$ is $\mathbf{S}$-free if no symbol $\mathbf{S}_{n}$ occurs in $\mathfrak{F}$.

3.11. Definition. Let $S_{0}, \ldots, S_{n}, \ldots$ be any sequence of sets and $\mathcal{M}^{*}=\mathcal{M}^{*}\left(S_{0}, \ldots, S_{n}, \ldots\right)$.

(i) Suppose $B, C_{1}, \ldots, C_{i-1} \in \mathcal{H}^{*}$, where $i \geqslant 1$. We say that $B$ is $\mathcal{M}^{*}$-dependent on $C_{1}, \ldots, C_{i-1}$ if for some $\mathbf{S}$-free formula $\mathfrak{F}\left(X, Y_{1}, \ldots, Y_{i-1}\right)$ of $\mathrm{L}^{*}$,

$B=$ the unique $X$ in $\mathbb{H}^{*}$ such that $F \mathcal{H} * \mathfrak{F}\left(X, C_{1}, \ldots, C_{i-1}\right)$.

(ii) $A$ (finite or infinite) sequence $B_{0}, \ldots, B_{n}, \ldots$ of elements of $\mathfrak{H}^{*}$ is said to be $\mathfrak{H}^{*}$-independent if for no $n, m_{1}, \ldots, m_{i-1}$ with $i \geqslant 1$ and $n \neq m_{1}, \ldots, m_{i-1}$ do we have $B_{n}$ is $\mathfrak{H}^{*}$-dependent on $B_{m_{1}}, \ldots, B_{m_{i-1}}$.

By using the results of this section, and following the lines of proof of 2.12 and the remark for 2.13 we can obtain the following.

3.12. THEOREM. Any generic sequence of sets $S_{0}, \ldots, S_{n}, \ldots(0 \leqslant n<\delta)$ is $\mathfrak{H}^{*}$-independent, where $\mathfrak{M}^{*}=\mathfrak{H}^{*}\left(S_{0}, \ldots, S_{n}, \ldots\right)$; the same holds true of the sequence of sets $S_{0}, \ldots, S_{n}, \ldots(0 \leqslant n<\omega)$ where $S_{n}=\{k:\langle k, n\rangle \in S\}$ $\delta=1, S$ is generic, and $\mathcal{H}^{*}=\mathcal{M}^{*}(S)$

To understand something of the significance of this result in more usual terms, we now prove some results relating the notion of $\mathcal{H}^{*}$-dependence to that of a set being hyperarithmetical in other sets. Following Kreisel [12], we make the following definition.

3.13. Defrnition. By an instance of the hyperarithmetic comprehension axiom we mean any closure of a formula of the form:

$$
\wedge \mathrm{x}[\mathrm{YY} \mathfrak{U}(\mathrm{x}, \mathrm{Y}) \leftrightarrow \wedge \mathrm{ZB}(\mathrm{x}, \mathrm{Z})] \rightarrow \bigvee \mathrm{X} \wedge \mathrm{x}[\mathrm{x} \in \mathrm{X} \leftrightarrow \vee \mathrm{Y} \mathfrak{U}(\mathrm{x}, \mathrm{Y})],
$$

where $\mathfrak{A}(\mathrm{x}, \mathrm{Y}), \mathfrak{B}(\mathrm{x}, \mathrm{Z})$ are arithmetical formulas (with possibly other free unranked variables).

Using 3.10 we now assume throughout the remainder of this section, that $S$ is a set hyperarithmetical in $O$ such that $S_{0}, \ldots, S_{n}, \ldots(0 \leqslant n<\delta)$ is a generic sequence, where each $S_{n}=\{k:\langle k, n\rangle \in S\}$. We take $\mathfrak{H}^{*}=\mathscr{M}^{*}\left(S_{0}, \ldots, S_{n}, \ldots\right)$.

3.14 Lemara. Suppose that $\mathfrak{F}(\mathrm{x}, \mathrm{Y})$ has only $\mathrm{x}, \mathrm{Y}$ free but that all bound 2nd order variables in $\mathfrak{F}$ are ranked. Suppose also that $1=\mathcal{H}^{*} \wedge \mathrm{x} \vee \mathrm{Y} \mathfrak{F}(\mathrm{x}, \mathrm{Y})$. Thne for some $a \in O_{1}, \models_{\mathcal{H}} \wedge \wedge \mathrm{x} \vee \mathrm{Y}^{a} \mathfrak{F}\left(\mathrm{x}, \mathrm{Y}^{a}\right)$.

Proof. Let $D=\operatorname{Diag}\left(S_{0}, \ldots, S_{m}, \ldots\right)$ and (by 3.6), pick $Q \subseteq D$ with $Q \Vdash \wedge \mathrm{x} \vee \mathrm{Y} F(\mathrm{x}, \mathrm{Y})$. Then by 3.3 (iii) and 3.1 (vii),

$$
(\nabla k)\left(\nabla Q^{\prime}\right)(\mathbb{H} e)\left\{Q^{\prime} \supseteq Q \Rightarrow e \in O_{1} \&\left(H Q^{\prime \prime}\right)\left[Q^{\prime \prime} \supseteq Q^{\prime} \& Q^{\prime \prime} \Vdash \vee \vee Y^{e} \mathfrak{F}\left(\bar{k}, \mathbf{Y}^{e}\right)\right]\right\} \text {. }
$$

The condition inside the brackets is $\prod_{1}^{1}$. Hence by Kreisel's result, given as (1) in the proof of 2.10, there exists a hyperarithmetical function $e=g\left(k, Q^{\prime}\right)$ which satisfies this condition for all $k$ and $Q^{\prime} \supseteq Q$ The range of $g$ on this set of $\left\langle k, Q^{\prime}\right\rangle$ is a hyperarithmetic subset of $O_{1}$. But then by Spector [16] this range is bounded above by some $a \in O_{1}$. It is seen from 3.1 (vi) that if $Q^{\prime \prime} \| V \mathrm{Y}^{e} \tilde{\xi}\left(\bar{k}, \mathrm{Y}^{e}\right)$ and $e \zeta a$ then also $Q^{\prime \prime} \| \vee \mathrm{Y}^{a} \mathfrak{F}\left(\bar{k}, \mathrm{Y}^{a}\right)$. Hence, for such $a$,

$$
(\nabla k)\left(\nabla Q^{\prime}\right)\left\{Q^{\prime} \supseteq Q \Rightarrow\left(\text { 过 } Q^{\prime \prime}\right)\left[Q^{\prime \prime} \supseteq Q^{\prime} \& Q^{\prime \prime} \mid-\vee Y^{a} \mathfrak{F}\left(\bar{k}, Y^{a}\right)\right]\right\},
$$

so that $Q \Vdash \wedge \mathrm{x} \vee \mathrm{Y}^{a} \mathfrak{F}\left(\mathrm{x}, \mathrm{Y}^{a}\right)$ and $\models \mathcal{M} * \wedge \mathrm{x} \vee \mathrm{Y}^{a} \mathfrak{F}\left(\mathrm{x}, \mathrm{Y}^{a}\right)$

3.15. THEOREM. Each instance of the hyperarithmetic comprehension axiom is true in $\mathfrak{K}^{*}$.

Proof. By definition of $\mathcal{H}^{*}$, it is sufficient to show that the result of substituting any ranked formulas for the free variables of the scheme in 3.13 gives a true sentence of $\mathcal{H}^{*}$. This is a consequence of the following: if $\mathfrak{F}_{1}(\mathrm{x}, \mathrm{Y}), \mathfrak{G}_{2}(\mathrm{x}, \mathrm{Y})$ are any two formulas with just $X, Y$ free, all of whose 2nd order variables are ranked, such that $=M_{*} \wedge \mathrm{x}\left[\bigvee \mathrm{Y} \mathfrak{G}_{1}(\mathrm{x}, \mathrm{Y}) \leftrightarrow \wedge \mathrm{Z} \mathfrak{G}_{2}(\mathrm{x}, \mathrm{Z})\right]$ then $F_{\mathcal{H}} * \bigvee \mathrm{X} \wedge \mathrm{x}[\mathrm{x} \in \mathrm{X} \leftrightarrow$ $\left.\leftrightarrow \bigvee \mathrm{Y} \mathfrak{F}_{1}(\mathrm{x}, \mathrm{Y})\right]$. To prove this, let $\widetilde{F}_{1}(\mathrm{x}, \mathrm{Y}, \mathrm{Z})=\left[\mathfrak{G}_{2}(\mathrm{x}, \mathrm{Z}) \rightarrow \mathfrak{G}_{1}(\mathrm{x}, \mathrm{Y})\right]$ and $\mathfrak{F}_{2}(\mathrm{x}, \mathrm{Y}, \mathrm{Z})=\left[\mathfrak{G}_{1}(\mathrm{x}, \mathrm{Y}) \rightarrow \mathfrak{G}_{2}(\mathrm{x}, \mathrm{Z})\right]$. Thus $=F_{\mathcal{H}} * \wedge \mathrm{x} \vee \mathrm{Y} \vee \mathfrak{Z F}_{1}(\mathrm{x}, \mathrm{Y}, \mathrm{Z})$ and $=\mu^{*} \wedge \mathrm{x} \wedge \mathrm{Y} \wedge \mathrm{Z} \mathfrak{\mho}_{2}(\mathrm{x}, \mathrm{Y}, \mathrm{Z})$. By 3.14 we can find $a \in O_{1}$ such that $=N^{*} \wedge \mathrm{x} \vee \mathrm{Y}^{a} \backslash \mathrm{Z}^{a} \mathfrak{F}_{1}\left(\mathrm{x}, \mathrm{Y}^{a}, \mathrm{Z}^{a}\right)$. Trivially, $=\mathcal{H}_{*} \wedge \mathrm{x} \wedge \mathrm{Y}^{a} \wedge \mathrm{Z}^{a} \mathfrak{F}_{2}\left(\mathrm{x}, \mathrm{Y}^{a}, \mathrm{Z}^{a}\right)$. It is thus seen that $=\mathcal{N} * \wedge x\left[\bigvee Y^{a}\left(_{1}\left(x, Y^{a}\right) \leftrightarrow \wedge Z^{a}\left(\mathfrak{F}_{2}\left(x, Z^{a}\right)\right]\right.\right.$. From this and the hypothesis, we obtain $\models_{\mathcal{H}} \wedge \mathrm{x}\left[\bigvee \mathrm{X} \mathfrak{G}_{1}(\mathrm{x}, \mathrm{Y}) \leftrightarrow \vee \mathrm{Y}^{a}\left(\mathfrak{b}_{1}\left(\mathrm{x}, \mathrm{Y}^{a}\right)\right]\right.$. But, again by definition of $\mathfrak{H}^{*}, \models \mathcal{H}^{*} \vee \mathrm{X} \wedge \mathrm{x}\left[\mathrm{x} \in \mathrm{X} \leftrightarrow \vee \mathrm{Y}^{a} \mathfrak{G}_{1}\left(\mathrm{x}, \mathrm{Y}^{a}\right)\right]$.

It can also be shown that $\mathcal{K}^{*}$ is a model of the $\sum_{1}^{1}$-axiom of choice (discussed by Kreisel [12]), but the proof is somewhat more involved.

3.16. THeOREM. Suppose $C_{1}, \ldots, C_{i-1} \in \mathcal{H}^{*}$, where $i \geqslant 1$. Then every set $B$ which is hyperarithmetical in $C_{1}, \ldots, C_{i-1}^{\dagger}$ also belongs to $\mathfrak{H}^{*}$.

Proof. By relativization of Kreisel ([11], p. 114), the collection of sets hyperarithmetical in $C_{1}, \ldots, C_{i-1}$ is the intersection of all collections which form $\omega$-models of the hyperarithmetic comprehension axiom and which contain $C_{1}, \ldots, C_{i-1}$.

3.17 THEOREM. (i) $S \in \mathcal{M}^{*}$.

(ii) $O \notin \mathcal{H}^{*} ; O$ is not hyperarithmetic in any $S_{0}, \ldots, S_{m}$.

Proof. (i) If $S \in \mathbb{H}^{*}$ then for some ranked $\mathfrak{F}\left(\mathrm{x}, \mathrm{Y}, \mathbf{S}_{0}, \ldots, \mathbf{S}_{m}\right)$ which contains only the symbols $S_{0}, \ldots, S_{m}$, we have for every $k, n$, $\langle k, n\rangle \in S \Longleftrightarrow F_{\mathcal{H}} * \widetilde{F}\left(\bar{k}, \bar{n}, \mathbf{S}_{0}, \ldots, \mathbf{S}_{m}\right)$. But then $\models_{\mathcal{H}} \wedge \wedge x\left[x \in \mathbf{S}_{m+1} \leftrightarrow\right.$ $\left.\leftrightarrow \mathfrak{F}\left(\mathrm{x}, \overline{m+1}, \mathbf{S}_{0}, \ldots, \mathbf{S}_{m}\right)\right]$, so that $S_{m+1}$ would be $\mathcal{H}^{*}$-dependent on $S_{0}, \ldots, S_{m}$, contrary to 3.12 . 
(ii) If $O \in \mathscr{H}^{*}$ then also $S$, being hyperarithmetic in $O$ by hypothesis, would be in $\mathcal{M}^{*}$, contrary to (i). We then apply 3.16 .

We can now clarify part of the content of 3.12 as follows.

3.18 THEOREM. $S_{0}, \ldots, S_{n}, \ldots$ are hyperarithmetically incomparable.

Proof. We show, as typical, that $S_{0}$ is not hyperarithmetic in $S_{1}, \ldots, S_{i-1}(i \geqslant 1)$. Consider the relativized set of ordinal notations $0^{S_{1}, \ldots, S_{i-1}}$. By 3.17 (ii) and Spector ([16], p. 160), $\omega_{1}^{S_{1}, \ldots, S_{i-1}}=\omega_{1}$. But then by Spector ([16], p. 159) for any set $B$ hyperarithmetic in $S_{1}, \ldots, S_{i-1}$ there is an $e \in O_{1}$ such that $B$ is $\left(S_{1}, \ldots, S_{i-1}\right)$-recursive in the (relativized) $H_{e}^{S_{1}, \ldots, S_{i-1}}$. Following Kleene $([10]$, p. 35), we can get for each $e \in O_{1}$ a formula $\mathfrak{F}_{e}\left(\mathrm{x}, \mathbf{S}_{1}, \ldots, \mathbf{S}_{i-1}\right)$ of rank $\preceq 2^{e}$ such that for any $k$, $k \in H_{e}^{S_{1}, \ldots, S_{i-1}} \Longleftrightarrow \models \mathcal{M}_{*} * \widetilde{F}_{e}\left(\bar{k}, \mathbf{S}_{1}, \ldots, \mathbf{S}_{i-1}\right)$. It follows that $B$ is $\mathcal{M}^{*}$-dependent on $S_{1}, \ldots, S_{i-1}$. In particular, if $S_{0}$ were hyperarithmetical in the sets $S_{1}, \ldots, S_{i-1}$ then $S_{0}$ would also be $\mathcal{H}^{*}$-dependent on these sets, contrary to 3.12 .

With a little more work it can be shown that, generally, if $C_{1}, \ldots, C_{i-1} \in \mathcal{M}^{*}$ and $B$ is hyperarithmetical in $C_{1}, \ldots, C_{i-1}$ then $B$ is $\mathbb{H}^{*}$-dependent on $C_{1}, \ldots, C_{i-1}$.

The existence of hyperarithmetically incomparable sets was first obtained by Spector [15] using a measure-theoretic argument. 3.17 (ii) and 3.18 give a little improvement (via 3.10), since they produce such sets which are of lower hyperdegree than $O$. In fact, by using the remark following 3.10 in a systematic way, we could also find a sequence of hyperarithmetically incomparable sets $S_{0}, \ldots, S_{n}, \ldots$ which are recursive in $O$, and hence of lower hyperdegree than $O$. Kreisel pointed out to us that this last statement can also be derived from Spector's result [15] by using Gandy's basis result [5].

4. We conclude with applications of these notions and methods to set theory. In this case, the basic language $\mathrm{L}$ is that of $\mathrm{Z}-\mathrm{F}$ (ZermeloFraenkel) set theory. Following Cohen ([2] or [3]), consider a denumerable model $\mathcal{H}$ of $\mathrm{Z}-\mathrm{F}$ which is a segment of the constructible sets of Gödel. Let $\alpha_{0}$ be the least ordinal not in $\mathcal{M}$. $\mathrm{L}^{*}=\mathrm{L}^{*}\left(\mathbf{S}_{0}, \ldots, \mathbf{S}_{n}, \ldots\right)$ can now be taken to be a transfinite ramified type theory, with both types and ranks ranging over ordinals $<\alpha_{0}$ (so $\beta_{0}=\alpha_{0}$ ). We can thus regard it as an extension of the language considered at the beginning of the preceding section. (We continue to use $x, y, z, \ldots$ to range over $\omega$ and $X, Y, Z, \ldots$ over subsets of $\omega$.) Now the members of $\mathcal{H}^{*}=\mathcal{H}^{*}\left(S_{0}, \ldots\right.$, $\left.S_{n}, \ldots\right) 0 \leqslant n<\delta$, are all defined by ranked formulas in the extended sense. Alternatively, and what is preferable if one is dealing with set theory alone, one can take $\mathrm{L}^{*}$ to be a ramified type-free language in a way which formally copies the relativization to sets $S_{0}, \ldots, S_{n}, \ldots$ of the definition of constructibility given in Gödel [7]. In any case, without going into details about the exact form of the syntax, we take it for granted that the definitions 2.1 of forcing, 2.2 of generic sequences, and 2.7 of transforms of formulas, can be extended to $\mathrm{L}^{*}$ in such a way that 2.3-2.6 and 2.8 continue to hold for the $\mathrm{L}^{*}$ and $\mathcal{M}^{*}$ considered here. We shall refer to the corresponding definitions and results by 4.1-4.8.

Note that the existence of a generic sequence of sets is established in 4.5 essentially as in 2.5, using the denumerability of $\alpha_{0}$ to give an enumeration of the sentences of $\mathrm{L}^{*}$. Throughout the remainder of this section we assume that $S_{0}, \ldots, S_{n}, \ldots(0 \leqslant n<\delta)$ is a generic sequence, $\mathcal{H}^{*}=\mathcal{M}^{*}\left(S_{0}, \ldots, S_{n}, \ldots\right)$ and $D=\operatorname{Diag}\left(S_{0}, \ldots, S_{n}, \ldots\right)$. In case $\delta=1$, we write $S=S_{0}$ and take $S^{(n)}=\{k:\langle k, n\rangle \in S\}$; the formal counterparts $\mathrm{S}$ and $\mathrm{S}^{(n)}$ are introduced in a similar way.

By adapting the arguments from Cohen's ([2] or [3]) to the present formulation the following can be shown.

4.9 Theonem. (i) $\mathcal{M}^{*}$ is a model of Z-F (the Zermelo-Eraenkel axioms).

(ii) If $\delta$ is finite, it is also a model of $\mathrm{AC}$ (the axiom of choice) and GCH (the generalized continuum hypothesis).

Concerning (ii) here, note that every element of $\mathcal{M}^{*}$ is constructible in less than $\alpha_{0}$ steps from $S_{0}, \ldots, S_{\delta-1}$.

Now the definition 3.11 of $\mathcal{M}^{*}$-dependence and $\mathcal{M}^{*}$-independence can be carried over exactly as it stands to the present context. The same holds true of the theorem 3.12. This leads to the following conclusion.

4.10. THEOREM. Suppose $\delta=1$.

(i) For any $n, m_{1}, \ldots, m_{i-1}$ with $n \neq m_{1}, \ldots, m_{i-1}$, there is no $\mathbf{S}$-free formula $\mathfrak{F}\left(\mathrm{X}, \mathrm{Y}_{1}, \ldots, \mathrm{Y}_{i-1}\right)$ of $\mathrm{L}^{*}$ such that $\mathcal{S}^{(n)}$ is the unique $X$ in $\mathcal{M}^{*}$ with $=\Re * \widetilde{J}\left(X, S^{\left(m_{1}\right)}, \ldots, S^{\left(m_{i-1}\right)}\right)$.

(ii) No $S^{(n)}$ is constructible from any finite number of other $S^{(m)}-s$ re lative to $\mathfrak{M}^{*}$.

In particular, (ii) extends the result in Cohen [2] that the axiom of constructibility is false in $\mathcal{H}^{*}(S)$ for suitable generic $S$. (i) is of interest with respect to the result in Addison ([1], p. 354), according to which if one assumes the axiom of constructibility, any two sets of natural numbers have comparable $\prod_{2}^{1}$ degrees. In contrast, 4.9 (ii) and 4.10 (i) show that it is consistent with $\mathrm{Z}-\mathrm{F}, \mathrm{AC}$, and $\mathrm{GCH}$ that there is a sequence $S^{(0)}, \ldots, S^{(n)}, \ldots$, no member of which is set-theoretically there is a sequence of any finite number of other members.

4.11 THEOREM. If $\delta=1$ there is no set-theoretically definable wellordering of the continuum in $\mathfrak{K}^{*}$.

Proof. What comes to the same thing, there is no formula $\mathfrak{F}(\mathrm{X}, \mathrm{Y})$ of $\mathrm{L}$ which establishes a well-ordering relation in the set of all subsets Fundamenta Mathematicae, T. LVI 
of $\omega$ in $\mathfrak{H}^{*}$. Suppose the contrary. We write $X \preceq Y$ instead of $\mid=\mathcal{H} * \mathfrak{F}(X, Y)$. We write $X \equiv Y$ if $X, Y$ differ at only finitely many places. According to hypothesis, the formalization of the following is true in $\mathbb{K}^{*}$ :

(1) $\Delta \subseteq \Sigma(\omega) \& A \neq \emptyset \Rightarrow(\mathbb{H} X)[X \in \Delta \&(\forall Y)(Y \in \Delta \Rightarrow X \underline{Y} Y)]\}$.

Here $\Sigma(\omega)$ denotes the set of all subsets of $\omega$. Now let

$$
\Delta=\left\{X: X \equiv S^{(n)} \text { for some } n\right\} \text {. }
$$

The set $\Delta$ is formally definable in $L^{*}$ by use of the symbol $\mathbf{S}$ By hypothesis, we can find some $n_{0}, k_{0}$ and $X_{0}$ such that $\left(\nabla k \geqslant k_{0}\right)$ $\left[k \in X_{0} \Leftrightarrow k \in S^{\left(n_{0}\right)}\right]$ and

(3)

$$
(\forall Y)\left(Y \in \Delta \Rightarrow X_{0} \preceq Y\right) \text {. }
$$

Now the formal definition of $X_{0}$ can also be given explicitly in terms of $\mathbf{S}$. Thus there is a certain sentence $\mathfrak{5}$ of $\mathrm{L}^{*}$ which formally expresses $(3)$ and such that $\mathfrak{C}$ is true in $\mathbb{H}^{*}$. But then, since $S$ is generic find $Q \subset D(=\operatorname{Diag}(S))$ such that $Q \Vdash-\mathfrak{F}$. Now let $l$ be a natural number such that neither $\left(\left\langle\overline{l, n_{0}}\right\rangle \in \mathbf{S}\right)$ nor $\left(\left\langle\overline{l, n_{0}}\right\rangle \in \mathbf{S}\right)$ belongs to $Q$, and that $l \geqslant k_{0}$. We shall define a certain transform $\tau$ for application of 4.8 (the extension of 2.8). Since $\delta=1$, we need only consider a function $\tau$ of one argument. We take $\tau(m)=1$ if $m \neq\left\langle l, n_{0}\right\rangle$ and $\tau\left(\left\langle l, n_{0}\right\rangle\right)=0$. By 4.8 and choice of $l, Q \| \tau(\mathfrak{G})$. Hence $\tau(\mathfrak{\zeta})$ is true in $\mathcal{H}^{*}$. Let $\widetilde{S}$ be such that

$$
m \in \widetilde{S} \Longleftrightarrow(\tau(m)=1 \& m \in S) \vee(\tau(m)=0 \& m \notin S),
$$

i.e. $\widetilde{S}$ contains $\left\langle l, n_{0}\right\rangle$ if and only if $S$ does not contain it, while otherwise $\widetilde{S}$ agrees with $S$. Similarly, take $\widetilde{X}_{0}$ to be such that $k \in \widetilde{X}_{0} \Longleftrightarrow\left(k \neq l \& k \in X_{0}\right) \vee\left(k=l \& l \xi X_{0}\right)$. Let $\widetilde{S}^{(n)}=\{k:\langle k, n\rangle \in \widetilde{S}\}$ and $\widetilde{J}=\left\{X: X=\widetilde{S}^{(n)}\right.$ for some $\left.n\right\}$. Then $\tau(\mathfrak{G})$ expresses that $\widetilde{X}_{0}$ is the least element of $\widetilde{\Delta}$ under $\preceq$. (Since $\mathfrak{F}(\mathbf{X}, \mathrm{Y})$ does not contain the symbol $\mathbf{S}$, it is unaffected by $\tau$.) But $\widetilde{\Delta}=\Delta$ and, by choice of $l, \widetilde{X}_{0} \in \Delta$ and $\widetilde{X}_{0} \neq X_{0}$. Hence $X_{0}, \widetilde{X}_{0}$ would be distinct least elements of $\Delta$ under $\zeta$, which is a contradiction.

Thus, from 4.9 (ii) we obtain: it is consistent with $\mathrm{Z}-\mathrm{F}, \mathrm{AC}$ and $\mathrm{GCH}$ that there is no set-theoretically definable well-ordering of the continuum. (That is, it is consistent to adjoin to these axioms the statement, for each formula $\mathfrak{F}$ of $\mathrm{L}$ with two free variables, which expresses that $\mathfrak{F}$ does not determine a well-ordering of the continuum.) This bears on questions dealt with by Myhill and Scott [13] ().

${ }^{(4)}$ The following result of Scott, which will appear in that paper, is of special interest in this connection: it is provable in $\mathrm{Z}-\mathrm{F}$ that there is a definable well-ordering $\Delta$ with field $\Gamma$ a subset of the continum, such that any other well-ordering $\Delta_{1}$ of this sort has field $\Gamma_{1} \subseteq \Gamma$. A simple explicit definition of this $\Delta$ can be given.
4.12. THEOREM. If $\delta=\omega$, the prime ideal theorem for Bootean algebras is false in $\mathfrak{H}^{*}$. In particular, every prime ideal in the algebra of all subsets of $\omega$ in $\mathbb{N}^{*}$ is principal.

Proof. Suppose $\Gamma \in \mathfrak{K}^{*}$, and that $\Gamma$ is a non-principal prime ideal in the algebra of subsets of $\omega$ in $\mathcal{M}^{*}$. Thus $\Gamma$ contains all finite sets. By definition of $\mathcal{H}^{*}$, there is a formula $\mathfrak{F}\left(\mathbf{X}, \mathbf{S}_{0}, \ldots, \mathbf{S}_{n}\right)$ of $\mathrm{L}^{*}$, containing only $\mathrm{X}$ free and only the constant symbols $\mathbf{S}_{0}, \ldots, \mathbf{S}_{n}$, such that for every $A \in \mathcal{M}^{*}$ with $A \subseteq \omega$,

$$
A \in T \Leftrightarrow A \text { satisfies } \mathbb{F}\left(\mathrm{X}, \mathrm{S}_{0}, \ldots, \mathrm{S}_{n}\right) \text { in } \mathfrak{M}^{*} \text {. }
$$

We shall show, by way of contradiction, that $S_{n+1} \dot{\epsilon} \Gamma$ and $\left(\omega-S_{n+1}\right) \notin \Gamma$. Suppose $S_{n+1} \in T$. Then $F_{\mathcal{M}} * \widetilde{S}\left(\mathbf{S}_{n+1}, \mathbf{S}_{0}, \ldots, \mathbf{S}_{n}\right)$, so that for some $Q \subseteq D$ $\left(=\operatorname{Diag}\left(S_{0}, \ldots, S_{n}, \ldots\right)\right)$,

$$
Q \Vdash \mathfrak{F}\left(\boldsymbol{S}_{n+1}, \boldsymbol{S}_{0}, \ldots, \boldsymbol{S}_{n}\right) .
$$

Let $k_{0}$ be chosen so that for all $k \geqslant k_{0}$, neither $\left(\bar{k} \in \mathbf{S}_{n+1}\right)$ nor $\left(\bar{k} \xi \mathbf{S}_{n+1}\right)$ belongs to $Q$. Then let $\tau(k, m)=1$ if $m \neq n+1$ or $m=n+1$ and $k<k_{0}$, $\tau(k, m)=0$ if $m=n+1$ and $k \geqslant k_{0}$. For any $m$, the set $S_{m}^{(t)}$ corresponding to $S_{m}$ under $\tau$ is identical with $S_{m}$ if $m \neq n+1$; however $S_{n+1}^{(\tau)}$ agrees with $S_{n+1}$ only for $k<k_{0}$ while it agrees with $\omega-S_{n+1}$ for $k \geqslant k_{0}$. Trivially, $\tau$ is arithmetically definable. Thus by $4.8(2.8)$, and choice of $k_{0}$, $Q \Vdash \tau\left(\widetilde{\mho}\left(\mathbf{S}_{n+1}, \mathbf{S}_{0}, \ldots, \mathbf{S}_{n}\right)\right)$, so the sentence $\tau\left(\widetilde{\mho}\left(\mathbf{S}_{n+1}, \mathbf{S}_{0}, \ldots, \mathbf{S}_{n}\right)\right)$ is true in $\mathcal{K}^{*}$. But, by (1) and definition of $\tau$, this sentence expresses that $\left[\left(S_{n+1} \cap k_{0}\right) \cup\left(\omega-S_{n+1}\right) \cap\left(\omega-k_{0}\right)\right] \in \Gamma$. Thus $\left(\omega-S_{n+1}\right) \cap\left(\omega-k_{0}\right) \in \Gamma$ since $\Gamma$ is an ideal and then $\left(\omega-S_{n+1}\right) \in \Gamma$ since all finite sets belong to $\Gamma$. But this contradicts $S_{n+1} \in \Gamma$, since $\Gamma$ is supposed to be a prime ideal. Similarly, we can show that $\omega-S_{n+1} \in \Gamma$ would lead to a contradiction.

By 4.9 (i) we see that the prime ideal theorem for Boolean algebras is independent of Z-F. This implies the result in Cohen's paper [2] that $\mathrm{AC}$ is independent of Z-F. Cohen's argument makes use of a special selection of the generic sets $S_{0}, \ldots, S_{n}, \ldots$ so as to introduce suitable symmetries into $\mathfrak{M}^{*}$. The preceding shows that this is not necessary. Even more directly, it is easy to show by the transform arguments given here that there is no choice set in $\mathbb{N}^{*}$ selecting reats from the cosets of the rationals in the reals $\left(\right.$ of $\left.\mathcal{M}^{*}\right)\left(^{5}\right)$. What comes to much the same thing, if we take $X \equiv Y$ (for $X, Y \subseteq \omega$ ) to mean that $X, Y$ differ at only a finite number of places, there is no choice set in $\mathfrak{K}^{*}$ selecting an element from each member of the collection $\Gamma$ of $\equiv$-classes (in $\left.M^{*}\right)$. Let $\Gamma_{1}$ be the collection (in $\mathcal{M}^{*}$ ) of pairs of the form $\{[X],[\omega-X]\}$, where

(5) Thus, one of the standard methods for obtaining the existence of Lebesgue on-measurable sets is not available in $1 \mathcal{C}^{*}$. Of course, this is hardly informative with respect to the interesting question whether the hypothesis that all sets of real numbers are Lebesgue measurable is consistent with "positive" measure theory. 
$[X]$ is the $\equiv$-class of $X$. Dana Scott pointed out to us that the transform arguments can also be used to show that there is no choice set in $\mathcal{M}^{*}$ which selects an element from each member of $\Gamma_{1}$. Thus the axiom of choice for unordered pairs is false in $\mathcal{M}^{*}$ (as also obtained in Cohen [2]), and hence the ordering theorem is false in $\mu^{*}$; Scott's observation also implies 4.12. Of course, the hypothesis $\delta=\omega$ is needed for all these results.

The arguments of this paper suggest that the properties of $\mathcal{M}^{*}\left(S_{0}, \ldots\right.$, $\left.S_{n}, \ldots\right)$ have little to do with the particular choice of $S_{0}, \ldots, S_{n}, \ldots$ other than to insure that the sequence is generic. In fact, the following theorem ( $\left.{ }^{6}\right)$ shows that any two such models which are determined by generic sequences of the same length $\delta$ share exactly the same true $\mathbf{S}$-free $\mathrm{L}^{*}$-statements, and hence are L-equivalent; this further emphasizes the "genericity" of generic sequences.

4.13. THEOREM. Suppose that $\mathfrak{F}$ is an $\mathrm{S}$-free sentence of $\mathrm{L}^{*}$. Then $=\Re * \widetilde{\Im} \Leftrightarrow \emptyset \mid \sim \sim \Re$.

Proof. If $\varnothing \| \sim \sim \widetilde{F}$ then, since $\emptyset \subseteq D \quad\left(=\operatorname{Diag}\left(S_{0}, \ldots, S_{n}, \ldots\right)\right)$, $F_{\mathcal{M}} * \sim \mathcal{F}$ and hence $\models_{\mathcal{M}} * \mathfrak{F}$. Suppose $\mid=\mathcal{M} * \mathfrak{F}$. Pick $Q \subseteq D$ with $Q \| \widetilde{Y}$. To show $\varnothing-\sim \sim \widetilde{F}$ we must show $(2.1$ (iv)) that there does not exist $Q^{\prime}$ with $Q^{\prime} \mid-\sim \widetilde{F}$. Suppose the contrary. We can then define a function $\tau$ which is 0 for only finitely many arguments and is sueh that $\tau\left(Q^{\prime}\right) \subseteq D$. Then $\tau\left(Q^{\prime}\right) \mid-\sim \tau(\mathscr{F})$ by 4.8. But, by hypothesis $\mathfrak{F}$ contains no $\mathbf{S}_{n}$, so $\tau(\mathfrak{F})=\widetilde{F}$. Hence $\tau\left(Q^{\prime}\right) \Vdash \sim \widetilde{F}$. Let $Q^{\prime \prime}=Q \cup \tau\left(Q^{\prime}\right)$. Then $Q^{\prime \prime} \subset D$ (and hence is consistent) and $Q^{\prime \prime} \|-\widetilde{F}$ and $Q^{\prime \prime} \| \sim \mathcal{F}$. This is impossible, so $\emptyset \mid \sim \sim \sim \widetilde{F}$.

It is also not difficult to see that for any two generic sequences of finite length the corresponding models $\mathscr{K}^{*}$ have exactly the same true $\mathbf{S}$-free $\mathrm{L}^{*}$ sentences. The striking difference in properties comes, as we have seen in this section, when we pass from $\delta$ finite to $\delta=\omega .4 .13$ is really a result of general character about the notion of forcing. We did not present it in $\S 2$, where it would have been trivial, or in $\S 3$, where it would have been more trouble to show that different properties are actually obtained for $\delta$ finite and $\delta=\omega$, for the special case studied there.

We conclude with several disparate remarks. First of all, while the main results of this section were phrased in model-theoretic terms, they can systematically be recast into finitist proofs of relative consistency of certain extensions of Z-F to Z-F, for example by the method outlined by Cohen in his paper [2] or [3], Part II. Second, it would be natural to expect that the gap between the work of $\S 3$ on hyperarithmetic

( $\left.{ }^{6}\right)$ This result was realized after the Symposium mentioned in footnote *. About the same time Azriel Léry independently found the same result, as well as an extre mely useful generalization of it. The latter will appear in some forthcoming work of his. analysis and that of $\S 4$ on set theory should be filled with applications to the full system of classical analysis of both 2nd and higher orders. While some applications can be found by extracting models of analysis from models of Z-F, we do not know what direct approach would be most successful. Finally, we have not given any consideration here to applications which involve imposing certain relationships in advance on a generic sequence or which involve introduction of constants of higher type-such as was done by Cohen [3] in his proof of the independence of GCH from Z-F and AC. Even without this, we feel the work here gives further indication of the great range of applicability and fruitfulness of the notions of forcing and generic sets.

\section{References}

[1] J. W. Addison, Some consequences of the axiom of constructibitity, Fund. Math., 46 (1959), pp. 337-357.

[2] Paul J. Cohen, The independence of the axiom of choice, Mimeographed notes, Mathematics Department, Stanford University (May, 1963), $32 \mathrm{pp}$.

[3] - The independence of the continuum hypothesis, Proc. Nat. Acad. of Sci. U.S.A., 50 (1963), pp. 1143-1148 (Part I) and 51 (1964), pp. 105-110 (Part II).

[4] S. Feferman and C. Spector, Incompleteness along paths in progressions of theories, Journ. Symbolic Logic. 27 (1962), pp. 383-390.

[5] R. Gandy, On a problem of Kleene's, Bull. Amer. Math. Soc. 66 (1960), pp. 501-502.

[6] - Proof of Mostowski's conjecture, Bull. Acad. Polon. Sci., Sér. math., 8 (1960), pp. 571-575.

[7] Kurt Gödel, Consistency-proof for the generalized continuum hypothesis, Proc. Nat. Acad. Sci. 25 (1939), pp. 220-224.

[8] - The consistency of the axiom of choice and the generalized continuum hypothesis with the axioms of set theory, Annals of Mathematics Studies No. 3, Princeton (1940), $66 \mathrm{pp}$

[9] S. C. Kleene, Hierarchies of number-theoretic predicates, Bull. Amer. Math. Soc. 61 (1955), pp. 193-213.

[10] - Quantification of number-theoretic functions, Compos. Math., 15 (1959), pp. 23-40.

[11] G. Kreisel, Set theoretic problems suggested by the notion of potential totality, Proc. Symp. Infinistic Methods, Warsaw 1961, pp. 103-140.

[12] - The axiom of choice and the class of hyperarithmetic functions, Indag. Math. 24 (1962), pp. 307-319.

[13] J. Myhill and D. Scott, Ordinal definability, in preparation.

[14] K. Schütte, Beweistheorie, Berlin 1960, 355 pp.

[15] C. Spector, Measure-theoretic construction of incomparable hyperdegrees, [15] C. Spector, Measure-theoretic const
Journ. Symbolic Logic 23 (1958), pp. 280-288.

[16] - Recursive well-orderings, Journ. Symbolic Logic 20 (1955), pp. 151-163. STANFORD UNIVERSITY

Reçu par la Rédaction le 20. Б. 1964 